\title{
Purple Staining of Archaeological Human Bone: An Investigation of Probable Cause and Implications for Other Tissues and Artifacts
}

\author{
Garrard Cole and Tony Waldron \\ Institute of Archaeology, University College London, 31-34 Gordon Square, London WC1H OPY, UK \\ Correspondence should be addressed to Garrard Cole; tcrngco@ucl.ac.uk
}

Received 6 January 2016; Accepted 3 May 2016

Academic Editor: Hugo Cardoso

Copyright (c) 2016 G. Cole and T. Waldron. This is an open access article distributed under the Creative Commons Attribution License, which permits unrestricted use, distribution, and reproduction in any medium, provided the original work is properly cited.

\begin{abstract}
Excavations in the 1990s at the medieval Chapter House of Worcester Cathedral, UK, revealed medieval human skeletal remains, some of which exhibited a distinctive purple coloration. The nature of the colored bone was investigated using solvents for stain extraction, scanning electron microscopy (SEM), energy dispersive X-ray spectroscopy (EDX), X-ray diffraction (XRD), X-ray fluorescence (XRF), plane polarized (PPL) and cross-polarized (XPL) light microscopy, and auto fluorescence (AF) microscopy. Normal bone from the cemetery was used as a control. The color does not arise from a stain soluble in normal organic solvents. EDX and XRD analysis showed no significant difference between purple and normal bone. XRF analysis shows the presence of trace levels of iron, manganese, zinc, and copper in the affected material. This exhibited a pink color in acid phase and a blue color in alkaline phase. These two states were reversible. The alkaline phase gradually changed irreversibly to yellow over time. These data suggest that the coloration is consistent with the presence of high levels of purple acid phosphatase (PAP) enzyme. The presence of trace amounts of iron, manganese, zinc, and copper suggests a plant or fungal origin for the putative PAP, possibly a member of the Aspergillus ficuum species.
\end{abstract}

\section{Introduction}

Excavations carried out in the 1990's at the circular Chapter House of Worcester Cathedral revealed a large number of inhumation burials, oriented east-west and generally lacking grave goods. Some graves had been truncated by the foundations of the Chapter House, indicating the cemetery predated construction of the building. Others were cut by later burials or other structures. The human remains probably date from $c$ $680 \mathrm{AD}$ to $\mathrm{c} 1100 \mathrm{AD}$, based on archaeological and historical evidence [1].

Some of the skeletal material exhibited a bright purple coloration (see Figure 1) affecting a number of skeletons located throughout the Chapter House. This coloration was noted on a variety of skeletal elements. It was not a surface phenomenon as the color penetrated the full thickness of the affected material. The lack of substantial areas of concentration argues against the hypothesis that the bone coloration was the result of dispersal of waste from some unknown dying process on the site. Similarly, the relatively uniform prevalence over all age and sex groups argues against a pathological process.

Archaeological bone rarely matches the normal color of natural human bone. Such matches occur only in rare circumstances of excellent preservation, such as undisturbed burial in graves directly cut into chalk. More typically, recovered inhumed archaeological bone exhibits various shades of brown, presumably due to Fe staining. This may vary from light superficial staining to complete discoloration in material recovered from waterlogged contexts, possibly arising from the inclusion of humic material [2] or burial in waterlogged silts [3]. Such environmental staining tends to be widespread, affecting whole elements, skeletons, and cemeteries. The presence of metallic grave goods in close proximity to bone may result in localized blue-green staining if copper alloy grave goods are present. Other workers have reported mixed red, black, and violet-blue stains [4]. A literature review of material from other archaeological sites revealed two sites 


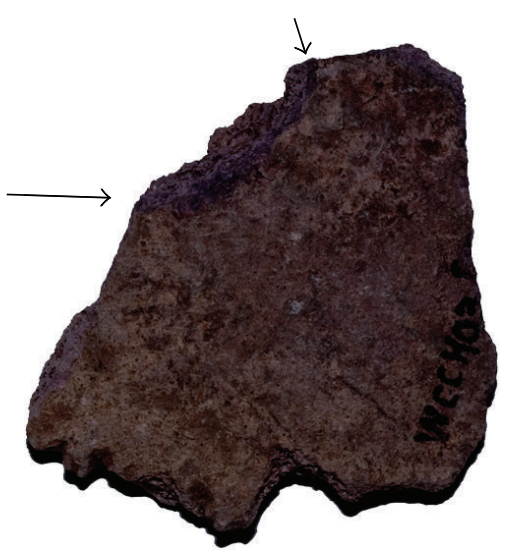

(a)

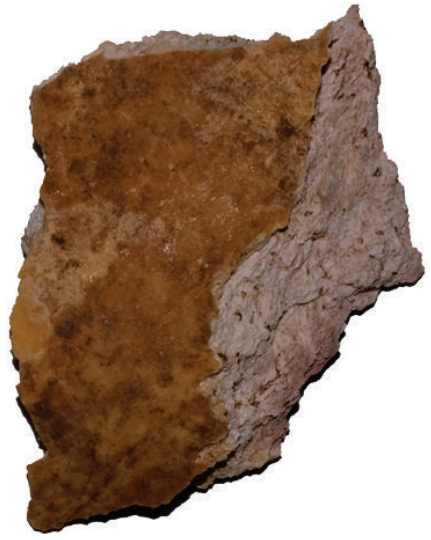

(b)

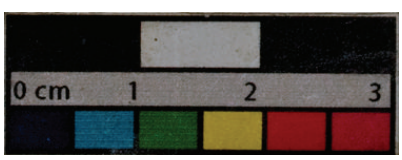

(c)

FIGURE 1: Samples of purple stained bone (a) and normal bone (b) taken from the parietal region of the respective crania. The bone samples both show the view of the outer table. The upper left of the purple stained sample and the lower right corner of the normal sample show cross sections through the diploe to the inner table. There is no variation of coloration through the full thickness of the purple stained specimen. This lack of variation makes it difficult to see the transition from outer table to diploe in photographs. The diploic zone lies between the two solid arrows. The normal specimen shows a typical variable intensity brownish color on the outer table. This color change is largely localized to the outer table. The diploe and inner table show much paler slight brownish tints differing from the natural white color of human bone.

with possibly comparable material. Isolated cases of localized pink or purple discoloration were observed on material at the Spitalfields postmedieval cemetery [5]. This coloration was ascribed to the effects of yeast fungus, based on the presence of fungal hyphae. More pink colored material was observed at the London Hospital Anatomy School cemetery [6]. The effects observed there are thought to reflect medical preservation practices carried out on the material.

Further coloration changes may arise in cremated bone resulting in specimens varying from orange, beige, light brown, dark brown, and black through gray and calcined pure white material. The variation in color is correlated with the cremation temperature, oxygen availability, burning environment, and duration of the cremation process [7-9]. Bright blue coloration of animal bone recovered from cave deposits is also thought to be the result of the presence of guano in the cave [10] or the result of burning [11].

Dobson [12] describes John Hunter's experiments feeding madder to animals, resulting in pink staining of developing bone. This phenomenon has been exploited to investigate growth mechanisms of bone [12-14]. Similar pink staining has been observed in human bone arising from the accumulation of alizarin, the pigment of madder [15]. Richter [16] also proposed that such pink staining could derive from the presence of purpurin carbonic acid.

Pink coloration is frequently observed in human teeth in forensic cases. Given the presence of iron compounds in such teeth, the coloration is thought to arise from seepage of hemoglobin or its decomposition derivatives after hemolysis [17]. Pink coloration has been observed in some teeth from individuals recovered from the medieval leper cemetery at St. James and Mary Magdalene, Chichester [18]. Dye and colleagues came to no conclusion over the origin of the coloration.

This literature review above focuses on color changes to bone primarily from an archaeological viewpoint. The problems relating to the formation of color changes in more complex forensic settings are covered comprehensively elsewhere $[19,20]$.

The aim of this paper was to investigate the vibrant nature of the purple coloration and the resultant structural differences and to identify possible causes. We planned to check whether the effect was the same as that reported in material from Spitalfields and London Hospital and whether it was the result of a pathological condition, of simple staining by likely stains such as indigo, of burning, or of fungal origin. In particular, we aimed to identify a precise mechanism to explain both the vivid coloration and the internal structural changes of the affected material. Finally, this knowledge would be used to look at the implications of similar changes in other nonosseous materials.

\section{Material and Methods}

2.1. Material. The excavation of the floor of the Chapter House at Worchester Cathedral resulted in the recovery of about 180 skeletons. The remains were well preserved and buried in a brownish gray sandy silt sediment. The soil was dry with a $\mathrm{pH}$ of 7.0-7.5. In some graves, evidence for coffin timber and textiles survived [1]. 


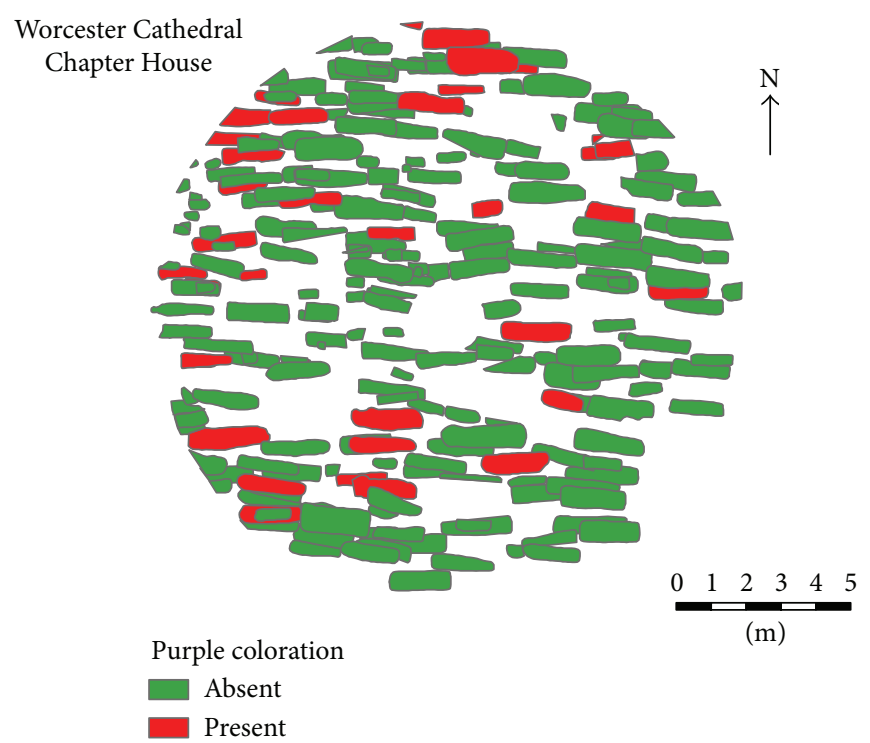

FIGURE 2: Worcester Cathedral Chapter House grave layout showing the distribution of burials distinguished by the presence of purple stained bone (red) versus those with only normal archaeological bone (green). The Chapter House is a circular structure, the foundations which have truncated some of the graves in a preexisting burial ground. The burials within the Chapter House would have been protected from subsequent taphonomic changes by the overlying built structure.

Some of the material exhibited a distinctive purple coloration over a greater or lesser part of the skeleton, whilst the remainder exhibited the typical brownish coloration commonly found on archaeological material. This coloration appeared to penetrate the full thickness of bone without variation in intensity or color. The distribution of affected and normal skeletons is shown in Figure 2.

Samples of purple and normal cranial bone were taken from skeletons selected at random in order to assess the nature and significance of the purple coloration. The normal bone was used as a control during the various analyses described below. Samples taken were of similar thickness from the parietal area of the cranium.

Both the case and control material exhibited normal endocranial and ectocranial morphology. For example, the vascular channels of the meningeal vessels were clearly visible in both samples. The normal material was well preserved and very robust requiring the use of a saw to cut suitably sized samples. In contrast, the purple material, whilst morphologically normal in appearance for the inner and outer tables, was structurally very weak. The samples could be crushed to powder between the fingers using moderate pressure. Small quantities of possibly fungal hyphae were noted on the surface of some affected bones. The material had already been cleaned of soil, so the extent of this fungal presence is unknown.

The curators of the human skeletal from the two sites $[5,6]$ with possibly similar material kindly provided access to the material in their care, allowing a comparison to be made visually. A small fragment of purple stained bone was taken to their archives allowing the respective curator and the lead author to make a simple visual check on the potentially similar material.
2.2. Analytical Methods. Spatial analysis was used to investigate the possibility of clustering of the affected skeletal material and to check for any demographic associations. This analysis was carried out using the ESRI ArcGIS 9.0 software package evaluating Global Moran's $I$ [21] and Local Moran's $I$ [22] spatial statistics. Moran's $I$ and its variants measure spatial autocorrelation, producing an $I$ value that is positive for clustered patterns, zero for random patterns, and negative for dispersed patterns. The process also calculates a $Z$ value, which is a measure of statistical significance of the patterning. $Z$ is a two-sided statistic so an absolute value of $Z$ greater than 1.96 corresponds to significance at the $5 \%$ level.

The specimens were also investigated using solvent extraction (to investigate possible stains), optical reflection and transmission microscopy, scanning electron microscopy (SEM), energy dispersive X-ray (EDX) spectroscopy, X-ray diffraction (XRD), X-ray fluorescence (XRF), and histological techniques. In all analyses, two samples were used: a control specimen with normal taphonomic changes and a purple colored test specimen. Archaeological material is frequently fragmented as a result of postdeposition taphonomic processes or poor excavation techniques. This effect was used to advantage here. Suitably sized fragments (about $7.5 \mathrm{~g}$ in weight) were selected for use in the various tests without resorting to making destructive sampling of otherwise intact material. One fragment was subsampled using a hand-saw to produce a specimen with a cut face of about $1 \mathrm{~cm}$ to be used in the SEM, EDX, and thin section tests. The remainder and other fragments were separately dried and powdered for use in the other analytic tests (see below).

Chloroform was used to test for the presence of indigo in complete specimens and extraction for other compounds was performed using a modification of Piepenbrink's [4] 
method. Piepenbrink used a 1:1 mixture of absolute ethanol and acetone in neutral, acidic, and alkaline phases. We tried a 1:1 mixture of IMS and acetone. Piepenbrink [4] specified the concentration of acid $(2 \mathrm{~N} \mathrm{HCl})$ and alkali $(2 \mathrm{~N} \mathrm{NaOH})$ but not the proportions of solid and solvent. We used $1 \mathrm{~g}$ on finely powdered bone, ground using a mortar and pestle, along with $1 \mathrm{~mL}$ of solvent. The solute was filtered using Whatman 42 filter paper to remove any fine suspended particles of powdered bone. The $\mathrm{pH}$ level was measured using a Hannah HI9024 $\mathrm{pH}$ meter calibrated at two points using buffers of $\mathrm{pH}$ of 4.0 and 7.0 .

Hitachi S3400 environmental SEM was used to analyze prepared and unprepared surfaces of case and control material. The unprepared material was a surface produced by a fine 60 tpi saw followed by sanding with 1200 grit emery paper. A photographic blower and fine brush was used to remove dust. The prepared material was similarly cut and then embedded in a clear Logitech 301 resin block. A vacuum pump was used to remove air bubbles and to allow the resin to penetrate the diploic space. The block was then cut using an Isomet low speed diamond saw and polished using $6 \mathrm{um}$ and $3 \mathrm{um}$ diamond polishing paste. The polished surface was then carbon coated. The analysis was carried out at $15 \mathrm{kV}$ acceleration voltage and variable pressure. The instrument was operated in both secondary electron and back-scattered electron modes.

Subsequently to SEM analysis, the polished specimens were used for thin section analysis. The carbon coat was first removed by polishing; then, the specimens were reduced to thin sections about 60 um thick using an Isomet diamond saw and polishing pastes as described above. The sections were mounted on standard glass slides using thin cyanoacrylate adhesive and covered with a thin coverslip. The thin sections were then assessed using a Leica DM EP microscope operating in plane polarized light (PPL) and cross-polarized light (XPL) modes. The software package QCapture Pro 6.0 was used to control the attached digital camera. A Leica Laborlux 12 POL microscope with a reflected U/V attachment was used to assess the sample for autofluorescence. This microscope was operated in reflected mode using a $420-490 \mathrm{~nm}$ exciting filter and a $520 \mathrm{~nm}$ suppression filter.

The EDX instrument attached to the SEM was used to initially assess the elemental spectrum of the control and case specimens. The method is not very sensitive and does not work for low mass elements. For this reason, the analysis was supplemented first by XRD analysis and then by XRF analysis to determine finer detail of the compound and elemental composition, respectively. The advantage of XRF analysis over XRD is that it is more sensitive and can detect low concentrations of elements in samples. The disadvantage is that it produces an elemental analysis rather than an assessment of compounds.

The XRD analysis was performed using a Bruker D4 Bragg-Brentano reflection geometry powder X-ray diffractometer. The copper source was operated at $40 \mathrm{kV}$ and $30 \mathrm{~mA}$. The scintillation counter output detector used a postsample graphite monochromator. Case and control samples weighing $5 \mathrm{~g}$ were reduced to powder and flat packed into welled silicon monolith holders. Each run was scanned from $5^{\circ}$ to $60^{\circ} 2 \Theta$ in steps of $0.05^{\circ}$ and using 4 seconds per step. Detected Xrays came from both $\mathrm{K} \alpha 1$ and $\mathrm{K} \alpha 2$. The resultant data were interpreted using PowderCell 2.4 to load and process reference spectra downloaded from the Chemical Database Service [23]. Analysis was carried out using reference spectra individually and in combination and in conjunction with the "optimising" option of PowderCell. The reference specimens included apatite, brushite, calcite, hydroxyapatite, purpurite, vivianite, and whitlockite, used singly and in combination.

The X-ray fluorescence analysis was carried out using a Spectro XLab 2000 energy dispersive XRF instrument. $5 \mathrm{~g}$ samples of case and control bone were dried at $60^{\circ} \mathrm{C}$ for 24 hours and then powdered in a percussive ball mill. The fine powdered bone was then dried for 24 hours at $110^{\circ} \mathrm{C}$ and pressed as $32 \mathrm{~mm}$ pellets using Hoechst wax binder at a ratio of $4 \mathrm{~g}$ sample to $1 \mathrm{~g}$ wax. The prepared pellets were analyzed in the XRF using a factory method that records the relative abundance of 48 elements as oxides. Three repeat measurements were made of each bone sample and an average was taken. The abundance of the major and minor elements was expressed as percentage weight (\%wt) and the trace elements were expressed as parts per million (ppm).

A simple presumptive test for the presence of PAP was made using Phosphatesmo KM test strips (Macherey-Nagel 906 07). Samples of $1 \mathrm{~g}$ powdered purple bone, $1 \mathrm{~g}$ powdered normal bone, and solvent were prepared and tested using the manufacturer's protocol.

\section{Results and Discussion}

3.1. Results. The global spatial analysis showed no significant patterning (Moran's $I=-0.01 Z=-0.29$ ) but the local variation suggests some limited clustering at three distinct areas of the northern margin of the cemetery (Figure 3). The red and green colors show statistically significant $(Z>1.96$ and $Z<-1.96$, resp., equivalent to a $5 \%$ significance level) clustering and dispersal. The demographic prevalence of the colored material is shown in Table 1. There appears to be no pattern, with all ages and sexes equally affected with an average prevalence of $22 \%$.

Color was not extracted by any of the organic solvents used. The purple bone powder turned pink in acid solution and blue in alkaline solution. This was observed when both water and organic solvents were used as the liquid phase. In the subsequent testing, distilled water alone was used. The neutral solution was colorless.

Using $0.2 \mathrm{M}$ acid and alkali, the filtered pink solution could be converted to colorless at neutral $\mathrm{pH}$ and blue at an alkaline $\mathrm{pH}$. Similarly, the blue filtrate could be converted to colorless at neutral $\mathrm{pH}$ and to pink at acidic $\mathrm{pH}$. The transition to pink occurred at about $\mathrm{pH} 6.0$, whilst the transition to blue occurred at about $\mathrm{pH} 10.0$. This cycle could be repeated numerous times, with the only change being a gradual diminution of intensity of pink or blue color with increasing acid/alkali quantities. However, once the alkali phase entered the yellow state, no change of state could be induced by variation of acidity or alkalinity.

Samples of the two colored solutions were left standing in the laboratory at room temperature and ambient lighting 
TABLE 1: Calculated prevalence values for the presence of colored bone classified according to broad age groups, biological sex where observable, and combined as a whole. The lower and upper levels of the respective $95 \%$ confidence intervals for the prevalence values are included to test the null hypothesis that there is no variation of prevalence across the various categories. The $95 \%$ confidence intervals for all the subgroups overlap indicating that the null hypothesis holds at the $5 \%$ significance level ( $N$ : number of individuals; CI: confidence interval).

\begin{tabular}{lcccccc}
\hline & Infant & Juvenile & Adult & Male & Female & Total \\
\hline$N$ purple & 4 & 10 & 7 & 11 & 6 & 38 \\
$N$ normal & 24 & 43 & 32 & 46 & 26 & 171 \\
Prevalence\% & 16.7 & 23.3 & 21.9 & $11.0-38.8$ & $13.9-42.1$ & $11.0-42.1$ \\
$95 \%$ CI & $7.6-35.9$ & $13.2-37.7$ & $16.6-29.0$ \\
\hline
\end{tabular}

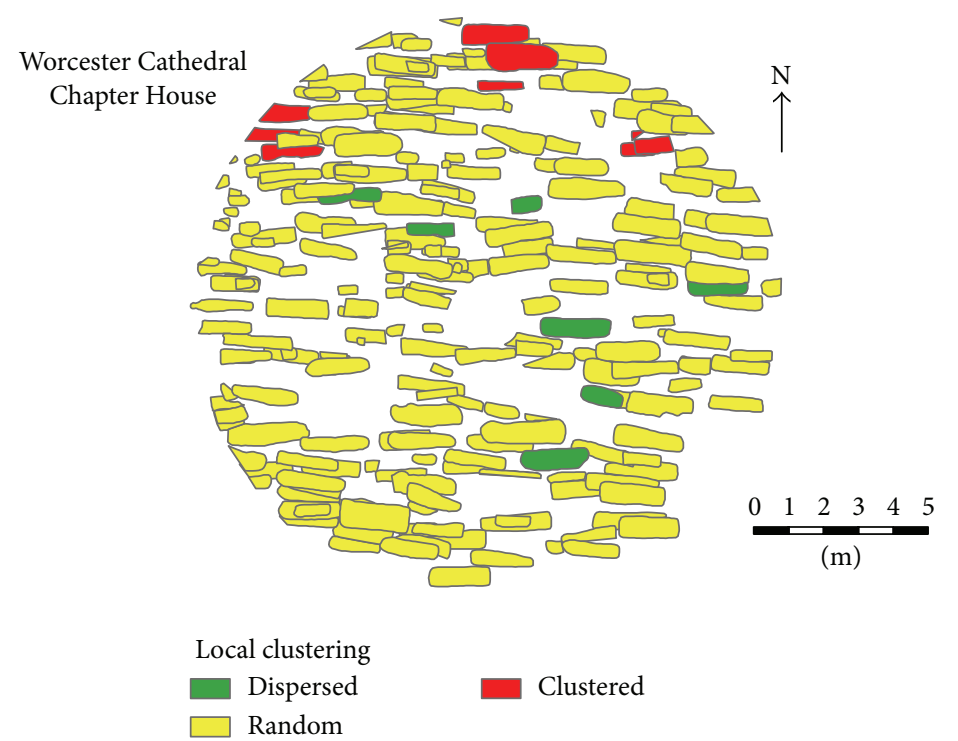

FIgURE 3: A plot of $Z$ value from the Anselin Local $I$ analysis of the spatial arrangement of burials shown in Figure 2. It shows statistically significant $(|Z|>1.965 \%$ level) clustered (marked by red) material around the northwest to northeast quadrants of the burials close to the Chapter House foundations and a more dispersed arrangement (marked by green) in the center and south to east quadrant. The clustered zone represents an above average concentration of purple stained bone and the dispersed zone represents a below average concentration assuming a random distribution (marked by yellow).

conditions. After about 10 days, the blue solution started losing color and by 18 days had turned to a distinct yellow color. The pink solution remained the same throughout (Figure 4). After several months, the color of the alkaline phase faded almost completely, and the bone powder had lost all color. The pink color of solid and solute was still present.

The results of the SEM analysis of the normal and purple bone are shown in Figure 5. The purple bone shows a distinct feathery appearance which occurs not only at the cut surface but also deep within the trabecular spaces. This suggests that it is not a consequence of the preparation process. The control specimen showed normal structure as expected. A filament with diameter of about $10 \mathrm{um}$, which might be a fungal hypha, was observed in one trabecular void of the prepared colored specimen, but no obvious fungal spores were observed.

The EDX results listed in Table 2 show no substantial difference in elemental composition. The major elements present are carbon, oxygen, phosphorus, and calcium. The calcium/phosphorus ratio is similar for both the purple bone and the normal bone. The results of the XRD analysis are

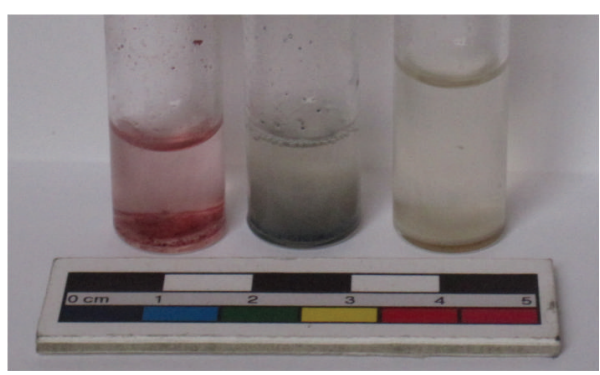

FIGURE 4: Coloration changes of powdered purple stained material in acid and alkaline phases at normal room temperature (about $20^{\circ} \mathrm{C}$ ). The three images show, from left to right, the resultant pinkish appearance after standing in acid phase for 10 days, a purplish appearance after standing in alkaline phase for 10 days, and a whitish appearance after standing in alkaline phase for 18 days. The pinkish and purplish states are reversible by increasing the alkalinity and acidity, respectively. The change of purplish appearance in the alkaline phase (center) to the whitish phase (right) is not reversible. The whitish appearance persists irrespective of changes to acid phase and back to alkaline phase. 


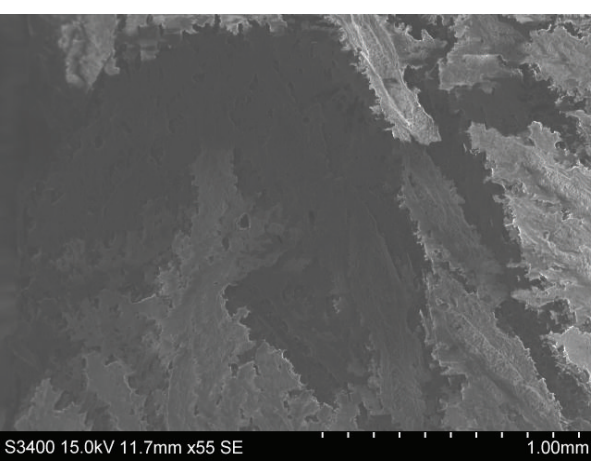

(a)

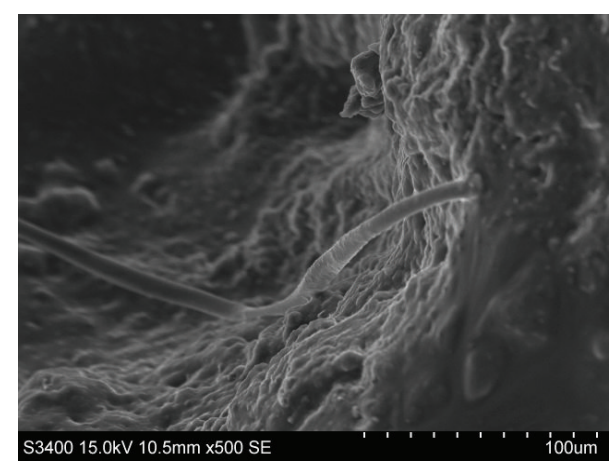

(b)

FIGURE 5: Secondary electron SEM analysis of the purple stained sample at low magnification $((a) \times 55)$ and moderate magnification $((b)$ $\times 500)$. Note the feathered appearance on the low magnification image (a) and possible fungal hyphae within one of the diploic voids in (b).

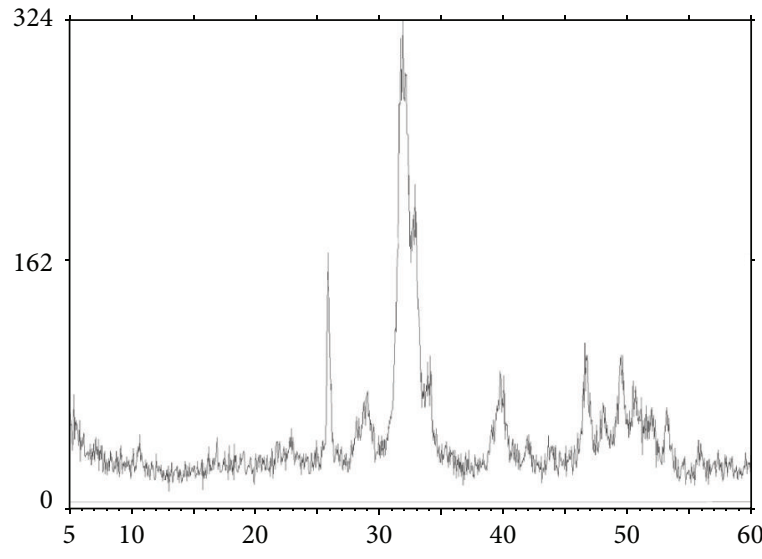

(a)

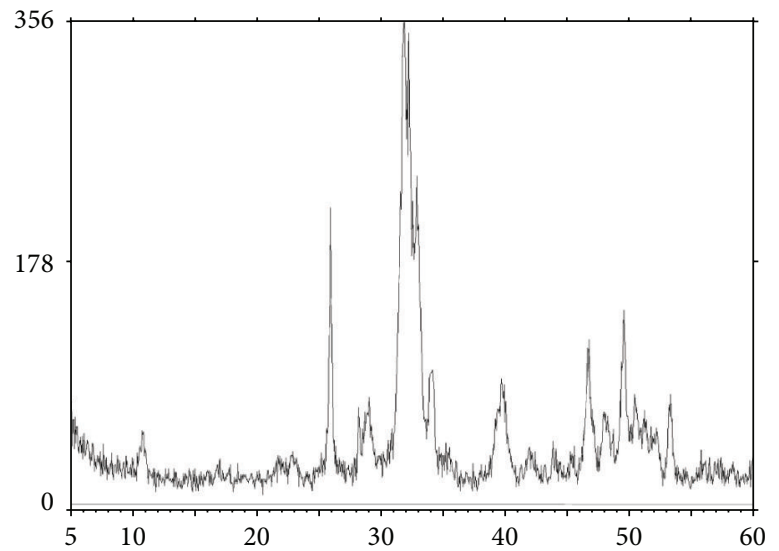

(b)

FiguRE 6: XRD results for normal (a) and purple stained (b) samples. The graphs both have detector count as the vertical scale and detector $2 \Theta$ angle (from $5^{\circ}$ to $60^{\circ} 2 \Theta$ in steps of $0.05^{\circ}$ ) as the horizontal scale. Hydroxyapatite is the best match in both cases.

TABLE 2: Hitachi SEM EDX results showing a crude indication of elemental composition. The high level of carbon in the data arises from the use of carbon coating on the prepared specimens. This was done in order to minimize charging effects on otherwise nonconducting specimens.

\begin{tabular}{lcc}
\hline Atomic\% & Normal & Purple \\
\hline $\mathrm{C}$ & 22.38 & 15.93 \\
$\mathrm{Ca}$ & 6.94 & 11.07 \\
$\mathrm{P}$ & 5.12 & 8.21 \\
$\mathrm{~S}$ & 0.27 & 0.10 \\
$\mathrm{Cl}$ & 0.00 & 0.42 \\
$\mathrm{Na}$ & 0.00 & 0.42 \\
$\mathrm{O}$ & 65.29 & 63.96 \\
$\mathrm{Ca} / \mathrm{P}$ ratio & 1.355 & 1.348 \\
\hline
\end{tabular}

shown in Figure 6. Again, no significant difference was observed. The best match is given by hydroxyapatite in both specimens.

The results of the XRF analysis are shown in Table 3. The main differences between the normal and purple specimens are higher proportions of the trace elements: manganese, iron, copper, and zinc. Interestingly, the levels of manganese and iron are higher by similar amount, about $250 \mathrm{ppm}$, in the purple bone with respect to the normal bone. As with the EDX data, the calcium/phosphorus ratio is lower in the purple bone than in the normal bone.

The thin section of sample normal bone showed typical birefringence under cross-polarized light. This was much reduced for the purple bone specimen (Figure 7). The internal structure of the distribution of Haversian canals was also less clear. The autofluorescence test showed a similar difference between normal and purple bone (Figure 8). The normal bone has regions of yellow and green colors indicating higher levels of autofluorescence than the purple bone which appears to be uniform muddy brown.

The Phosphatesmo KM test for the purple bone specimen resulted in a pinkish-brown stain that developed slowly over several minutes. The normal bone and solvent-only samples produced no observable response.

3.2. Discussion. Excavations carried out in the 1990's at the circular Chapter House of Worcester Cathedral revealed 
TABLE 3: Powder XRF results showing dominant elemental components. Bold is used to emphasize elements with a significant difference in average values between normal and purple colored specimens. There is no overlap of the $95 \%$ confidence intervals of the ppm value for any of the elements marked in bold. The null hypothesis that there is no difference in concentration of the respective elements in the normal and purple colored bone therefore does not hold at the 5\% significance level (av: average of 3 readings; sd: standard deviation; all units: ppm).

\begin{tabular}{|c|c|c|c|c|c|c|c|c|c|}
\hline Element & $\mathrm{Na}$ & $\mathrm{Si}$ & $\mathrm{P}$ & $S$ & $\mathrm{Ca}$ & $\mathrm{Mn}$ & $\mathrm{Fe}$ & $\mathrm{Cu}$ & $\mathrm{Zn}$ \\
\hline Normal av & 8700 & 2563 & 239233 & 4311 & 420533 & 48 & 665 & 21 & 147 \\
\hline Normal sd & 2390 & 57 & 451 & 117 & 1756 & 6 & 5 & 4 & 5 \\
\hline Purple av & 12967 & 2553 & 278933 & 75 & 450600 & 272 & 921 & 62 & 213 \\
\hline Purple sd & 1704 & 45 & 737 & 0 & 1058 & 21 & 26 & 3 & 3 \\
\hline
\end{tabular}

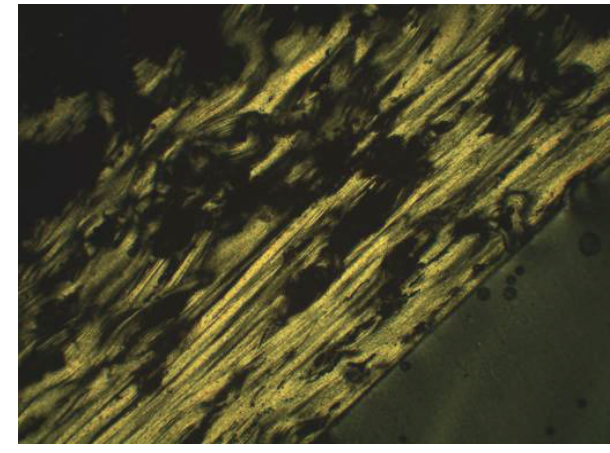

(a)

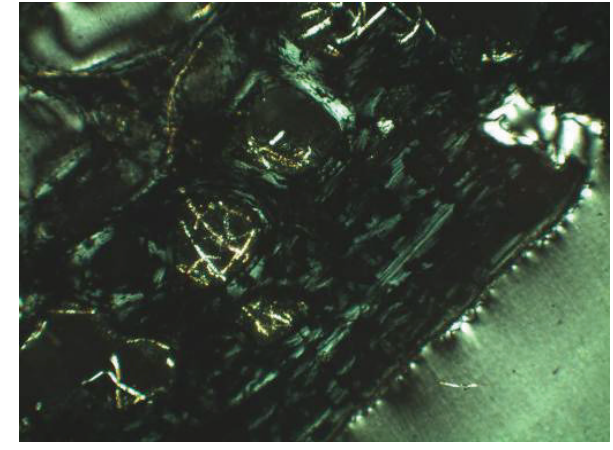

(b)

FIGURE 7: XPL images for normal (a) and purple stained (b) samples at equivalent stages of the extinction cycle. The normal sample shows a more ordered structure than the purple stained sample, along with a typical birefringence pattern. The extinction zones in the purple stained sample are very disorganized. The birefringence pattern is very disordered. There is also evidence of taphonomic cracking within the section. This indicates that the process responsible for unusual coloration affects the internal structure of the bone without affecting the gross morphology.

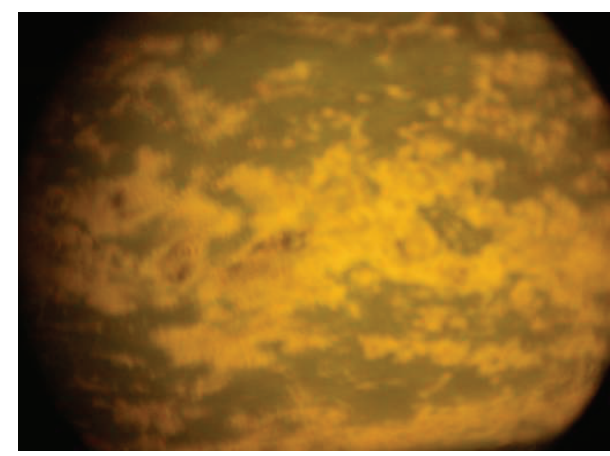

(a)

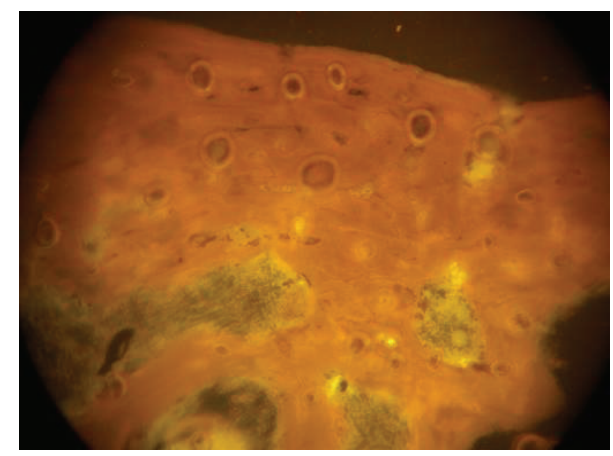

(b)

Figure 8: Autofluorescence imagery for normal (a) and purple stained (b) samples. The lighter greenish areas in the purple specimen are trabecular voids. The normal bone specimen appears to contain some residual collagen. There appears to be no or very little residual collagen in the purple stained sample.

a large number of inhumation burials, oriented east-west and generally lacking grave goods. Some graves had been truncated by the foundations of the Chapter House, indicating the cemetery predated construction of the building. Others were cut by later burials or other structures [1]. Some of the skeletal material exhibited a bright purple coloration affecting a number of skeletons located throughout the Chapter House. This coloration was noted on a variety of skeletal elements. The lack of substantial areas of concentration argues against the hypothesis that the bone coloration was the result of dispersal of waste from some unknown dying process. Similarly, the relatively uniform prevalence over all age and sex groups argues against a pathological process.

Comparative material from Spitalfields and London Hospital described as exhibiting a pink or purple coloration was examined by the lead author and respective material curator, using a small fragment of purple colored bone as a reference. The effect observed on the Worcester material is totally different from that observed on this material. In both cases, the coloration appears to be purely a surface effect with no significant penetration. The affected zones also have clear circumscribed boundaries. There is also no evidence of any 
bone degradation associated with the stained regions. The colors observed on the Spitalfields material are mixtures of red and black, possibly comparable to the effects observed by Piepenbrink. The changes observed on the Royal London material appear to be consistent with the use of anatomical preparation techniques used to make casts of blood vessels.

The vibrant nature of the coloration suggested several possible causes, such as simple staining by suitable compounds (indigo in this case) or partial cremation. A specific solvent test for indigo gave a negative result, allowing that mechanism to be excluded.

Cremation was considered as another potential mechanism. Cremated human bone changes color according to the exposure temperature and duration of cremation. The appearance of the affected bone bears a superficial similarity to cremated bone. Cremation commonly introduces morphological changes such as recrystallization, shrinkage, cracking, and occasional enlargement. A visual examination of the distribution of purple colored material in relation to normal suggested that cremation was an unlikely cause. Purple colored areas of bone were found interspersed among normal bone and tended preferentially to affect the skull (especially the skull base), the axial skeleton, and bones with a high concentration of trabecular bone (such as the femoral head). Whilst such a distribution argues against burning as a cause of the changes observed, XRD analysis was used to determine a definitive answer. The XRD spectra for normal and purple colored bone are similar. This alone definitely excludes burning as a cause of the purple coloration.

In addition to the vivid coloration, the purple colored material was also structurally modified. The cranial fragments could be crushed under strong finger pressure, whilst the normal samples were crushed only with difficulty using a pestle and mortar. The endocranial surface appeared normal with normal vascular track for the middle meningeal vessels. Brushite has been reported clinically in degenerative bone lesions associated with intervertebral disk disease CPPD [24]. It is also formed in space-consuming ways resulting in the mechanical destruction of bone [25]. However, brushite can be excluded as a possible cause. Whilst hydroxyapatite may be converted to brushite in an acid environment, the reported neutral $\mathrm{pH}$ of the local soil mentioned above argues against conversion to brushite as a causative mechanism as does the apparently normal gross morphology. The $\mathrm{XRD}$ analysis gives definitive evidence that brushite is not present.

SEM was used to examine the material for evidence of a possible cause of this weakness. There was no evidence for osteoclastic activity, which is usually marked by the presence of erosion tracks about 50 um wide [26]. This suggests a nonpathological cause for the weak structure of the purple bone.

Autofluorescence in bone is thought to reflect collagen content [27] or simply the presence of phosphates in archaeological contexts [28]. Prentice notes that autofluorescence is poor in young individuals and is reduced in regions of high bone turnover. The autofluorescence is seen as a strong greenish yellow response when specimens are illuminated at blue excitation [29]. The control specimen exhibits such a strong green yellow response, whilst the colored specimen displays a relatively uniform muddy brown response. Given that the XRD data shows similar calcium and phosphorus components, this suggests that there may be a significantly lower level of collagen in the colored material.

In a review of the origin of color in minerals, Nassau [30] describes a metal-metal charge transfer mechanism capable of producing vivid colors in minerals with appropriate trace impurities. For example, the characteristic deep blue color of hydrated iron phosphate vivianite arises from charge transfer between $\mathrm{Fe}^{2+}$ and $\mathrm{Fe}^{3+}$ following conversion of some of the $\mathrm{Fe}^{2+}$ to $\mathrm{Fe}^{3+}$ by oxidation in air. This mineral is found in archaeological contexts, requiring sources of iron, phosphate, and water in the presence of low levels of oxygen and sulfur [31]. This would not explain the pink coloration seen, so the presence of vivianite can be discounted as an explanation.

A nonmineral source of purple coloration is exhibited by the tartrate resistant acid phosphatase (TRAP) enzyme. This enzyme family is also known as purple acid phosphatase (PAP) [32]. These are cationic glycoproteins containing iron, which can show a characteristic purple color when isolated and concentrated [33].

The biochemistry of PAPs has been extensively reviewed (e.g., see $[34,35]$ ) and is briefly summarized as follows. They are a group of enzymes known as binuclear metalloenzymes with two closely spaced metallic ions forming the reactive binuclear center. They all exhibit a characteristic purple color resulting from a charge transfer transition between tyrosine and a metallic ion in the 3+ state. Examples of PAPs are found in plants, mammals, and some bacteria. The mammalian variants have a low molecular weight $(\sim 35 \mathrm{kDa})$ with an $\mathrm{Fe}^{3+}$ $\mathrm{Fe}^{3+} / \mathrm{Fe}^{2+}$ core forming a type of Haber-Wess-Fenton reactive center. PAPs from plants have a higher molecular weight $(\sim 55 \mathrm{kDa})$. The genetic sequences of mammalian variants are identical over about $80 \%$ of the genome. The binuclear core is variable according to species. In some plant species, the core is $\mathrm{Fe}^{3+}-\mathrm{Zn}^{2+}$ and in others it is $\mathrm{Fe}^{3+}-\mathrm{Mn}^{2+}$. PAPs have been detected in Aspergillus ficuum demonstrating a high molecular weight $(\sim 85 \mathrm{kDa})$ and a form closer to plant PAP rather than mammalian PAP. The gene encoding potential for PAP has also been found in a few other bacteria such as the cyanobacteria Synechocystis sp. and the mycobacteria Mycobacterium tuberculosis and $M$. leprae. In mammals, the primary functions of PAPs appear to relate to bone resorption, the generation of reactive oxygen species as part of the immune response, and iron transport through the body. In plants, the primary function is thought to relate to liberation of phosphates from the environment. The association of mammalian species of PAP with bone resorption has led to research into the development of PAP as a potential antiosteoporotic agent.

The distinctive variation in color of the acidic and alkaline states of the purple bone sample has been reported by others investigating TRAP [36]. TRAP with pink coloration in the active state and purple coloration in the inactive state eventually reduced to a yellow inactive state when incubated at $37^{\circ} \mathrm{C}$. Once in this yellow state, the material could not be reactivated [36]. This effect was noted in the purple material from Worcester. 
The Phosphatase KM test strip exploits a reaction between the PAP and the detection agent (typically sodium naphthyl phosphate and Brentamine Fast Blue B) which produces a vivid purple stain within a few seconds for fresh samples. More degraded stains produce a fainter indication after a longer period. Nonhuman PAP sources tend to produce a pink/brown response [37]. The slow response time and pinky/ brown stain generated by the purple bone specimen suggest that a degraded nonhuman PAP is present in the purple bone.

Overall, PAP seems to be a likely cause as its presence explains both the coloration changes and the structural changes. The potential PAP mechanism may give insights into the controversial issue of "pink teeth" which are found in both forensic and archaeological contexts. Camps [38] ascribed the coloration observed in the teeth of strangulation victims in the Christie case to pressure of strangulation forcing hemoglobin degradation products into dentine tubules. Such dental coloration has been observed in cases with other causes of death such as drowning [39], experimentally in rats [40] and even in living individuals [41], so the current consensus is that the effect is of unknown etiology.

In his report, Camps [38] noted that reversion spectroscopy suggested that the compound assumed to be responsible for the coloration was an unidentified "haem" compound not matching hemoglobin or its known degradation products. He also notes that the color observed in a victim of drowning had a more purple colored tint. Further work on pink teeth in forensic settings reinforces the idea that the coloration arises from the presence on a compound containing iron, assumed to be a degradation product of hemoglobin (see $[39,42-44])$. The work of Dye and colleagues [18] forms an interesting case study. They observed pink coloration in the teeth of a number of individuals from the medieval cemetery of St. James and Mary Magdalene, Chichester. Skeletal evidence demonstrated the presence of both tuberculosis and leprosy in the population buried in this cemetery. Schenk and colleagues [34] noted that mycobacteria appear to have the genetic components allowing for the synthesis of purple acid phosphatase. Given that PAP is known to contain iron, it would be a useful follow-up to see if there is an evidence for the presence of PAP in the Chichester teeth. Unfortunately, the authors could not track down the specimens used by Dye and colleagues, so this forms an avenue for further work.

Very similar purple coloration of organic material has been noted in conservation contexts (see $[45,46]$ ). Quandt [46] reports the presence of purple stains on some of the pages of the Archimedes Palimpsest. The report refers to the presence of bacterial spore chains and fungal structures. It was hypothesized that these organisms were responsible for both the coloration and fragile spongy nature of the affected areas. The pigments were sensitive to $\mathrm{pH}$ levels, changing from red through violet to blue as the $\mathrm{pH}$ was changed from acidic levels to alkaline levels. The stains are also described as penetrating completely through an affected folio. The coloration and degradation effects seem very similar to those observed in the purple bone from Worcester. If the underlying mechanism for the degradation of the palimpsest parchment is an acid phosphatase, that knowledge may open routes to improved conservation of the documents.

\section{Conclusions}

Some of the human skeletal remains recovered from excavations in the 1990's at the medieval Chapter House of Worcester Cathedral had a distinctive purple coloration. This coloration appeared to penetrate evenly the full thickness of bone.

A structured sequence of tests was used to investigate possible causes for the stain. These included demographic analysis to investigate potential patterning amongst affected individuals, solvent tests for likely stains, SEM and optical microscopy tests to investigate morphological and structural changes, EDX and XRD for compound analysis, and XRF for elemental analysis.

The purple coloration is not the result of a stain soluble in normal organic solvents. In addition to the general purple coloration, there is a widespread modification to the bone resulting in a loss of structural integrity and strength, whilst preserving normal morphology. EDX analysis showed no significant difference in composition for the affected and normal bone samples. Subsequent more detailed XRD analysis shows that both normal bone and the purple bone are composed of hydroxyapatite, with no contribution from brushite or whitlockite. Finally, XRF analysis revealed subtle differences between the affected and normal bone samples. It revealed the presence of trace levels of iron, manganese, zinc, and copper. The material exhibited a pink color in acid phase and a blue color in alkaline phase. These two states were reversible, one to the other. The blue color in the alkaline phase gradually and irreversibly changed to yellow over time.

These data suggest that the coloration is consistent with the presence of high levels of purple acid phosphatase enzyme. The color is the result of a charge transition between tyrosine and $\mathrm{Fe}^{3+}$ within the bone. The material also contains trace amounts of manganese, zinc, and copper. This suggests a plant or fungal origin for the putative PAP, possibly a member of the Aspergillus ficuum species. It is intriguing that such a dramatic and vibrant coloration of material appears to result from the presence of trace levels of specific elements, rather than some bulk staining effect. This knowledge about the mechanism underlying the purple coloration may have implications for the investigation of similar color changes observed in other classes of material, such as the parchment discussed above. It may also give insights into the origin of nonhaem $\mathrm{Fe}$ compounds reported to be present in "pink teeth."

\section{Competing Interests}

The authors declare that there are no competing interests regarding the publication of this paper.

\section{Acknowledgments}

The authors would also like to thank Christopher Guy for useful discussions on the original archaeological context of the Worcester material. The following colleagues from UCL Institute of Archaeology helped with the various tests: Sandra Bond (embedding specimens), Richard Macphail (AF), 
Patrick Quinn (XRF and thin sections), and Kevin Reeves (SEM/EDX). Margaret Clegg (Natural History Museum) and Don Walker (Museum of London Archaeology) provided access to and useful discussions of the comparative material in their collections. Finally, Martin Vickers (UCL Chemistry) helped with the XRD analysis.

\section{References}

[1] G. Guy, "An Anglo-Saxon cemetery at worcester cathedral," in Burial in later Anglo-Saxon England c. 650-1100 AD, J. Buckberry and A. Cherryson, Eds., pp. 73-82, Oxbow, Oxford, UK, 2010.

[2] M. M. E. Jans, H. Kars, C. M. Nielsen-Marsh et al., "In situ preservation of archaeological bone: a histological study within a multidisciplinary approach," Archaeometry, vol. 44, no. 3, pp. 343-352, 2002.

[3] Y. H. Edwards, A. Weisskopf, and D. Hamliton, "Age, taphonomic history and mode of deposition of human skulls in the River Thames," Transactions of the London and Middlesex Archaeological Society, vol. 60, pp. 35-51, 2009.

[4] H. Piepenbrink, "Two examples of biogenous dead bone decomposition and their consequences for taphonomic interpretation," Journal of Archaeological Science, vol. 13, no. 5, pp. 417-430, 1986.

[5] T. Molleson, M. Cox, H. A. Waldron, and D. K. Whittaker, The Spitalfields Project. Vol. 2: The Anthropology. The Middling Sort, Council for British Archaeology, York, UK, 1993.

[6] L. Fowler and N. Powers, Doctors, Dissection and Resurrection Men: Excavations in the 19th Century Burial Ground of the London Hospital, 2006, Museum of London Archaeology, London, UK, 2012.

[7] P. Shipman, G. Foster, and M. Schoeninger, "Burnt bones and teeth: an experimental study of color, morphology, crystal structure and shrinkage," Journal of Archaeological Science, vol. 11, no. 4, pp. 307-325, 1984.

[8] P. L. Walker, K. W. P. Miller, and R. Richman, "Time, temperature and oxygen availability: an experimental study of the effects of environmental conditions on the color and organic content of cremated bone," in The Analysis of Burned Human Remains, C. W. Schmidt and S. A. Symes, Eds., pp. 129-137, Academic Press, London, UK, 2008.

[9] C. Wells, "A study of cremation," Antiquity, vol. 34, no. 133, pp. 29-37, 1960.

[10] J. Robles, J. Arroyo-Cabrales, E. Johnson, B. L. Allen, and G. Izquierdo, "Blue bone analyses as a contribution to the study of bone taphonomy in San Josecito Cave, Nuevo Leon, Mexico," Journal of Cave and Karst Studies, vol. 64, no. 2, pp. 145-149, 2002.

[11] C. Chadefaux, C. Vignaud, E. Chalmin et al., "Color origin and heat evidence of paleontological bones: case study of blue and gray bones from San Josecito cave, Mexico," American Mineralogist, vol. 94, no. 1, pp. 27-33, 2009.

[12] J. Dobson, "Pioneers of Osteogeny: John Hunter: 1728-1793," Journal of Bone and Joint Surgery, vol. 30B, pp. 361-364, 1948.

[13] J. Belchier, "An account of the bones of animals being changed to a red colour by aliment only. By John Belchier, Surgeon, F. R. S," Philosophical Transactions, vol. 39, pp. 287-288, 1753.

[14] J. C. Brash, "Some problems in the growth and developmental mechanics of bone," Edinburgh Medical Journal, vol. 41, pp. 363387, 1934 .
[15] S. H. Steckoll, Z. Goffer, H. Nathan, and N. Haas, "Red-stained human bones from Qumran," Israel Journal of Medical Sciences, vol. 7, no. 11, pp. 1219-1223, 1971.

[16] D. Richter, "Vital staining of bones with madder," Biochemical Journal, vol. 31, no. 4, pp. 591-595, 1937.

[17] T. K. Dumser and M. Türkay, "Postmortem changes of human bodies on the bathyal sea floor-two cases of aircraft accidents above the open sea," Journal of Forensic Sciences, vol. 53, no. 5, pp. 1049-1052, 2008.

[18] T. J. Dye, D. Lucy, and A. M. Pollard, "The occurrence and implications of post-mortem 'pink teeth' in forensic and archaeological cases," International Journal of Osteoarchaeology, vol. 5, no. 4, pp. 339-348, 1995.

[19] T. L. Dupras and J. J. Schultz, "Taphonomic bone staining and color changes in forensic contexts," in Manual of Forensic Taphonomy, J. T. Pokines and S. A. Symes, Eds., pp. 315-340, CRC Press, Boca Raton, Fla, USA, 2014.

[20] J. T. Pokines and J. E. Baker, "Effects of Burial Environment on Osseous Remains," in Manual of Forensic Taphonomy, J. T. Pokines and S. A. Symes, Eds., pp. 73-114, CRC Press, Boca Raton, Fla, USA, 2014.

[21] P. A. Moran, "Notes on continuous stochastic phenomena," Biometrika, vol. 37, pp. 17-23, 1950.

[22] L. Anselin, "Local indicators of spatial association-LISA," Geographical Analysis, vol. 27, no. 2, pp. 93-115, 1995.

[23] CDS, “ICSD Web Access," https://cds.dl.ac.uk/.

[24] R. S. Lee, M. V. Kayser, and S. Y. Ali, "Calcium phosphate microcrystal deposition in the human intervertebral disc," Journal of Anatomy, vol. 208, no. 1, pp. 13-19, 2006.

[25] B. Herrmann and H. Newesely, "Dekompositionsvorgänge des Knochens unter langer Liegezeit 1. Die mineralische Phase," Anthropologischer Anzeiger, vol. 40, pp. 19-31, 1982.

[26] J. M. W. Quinn, S. Neale, Y. Fujikawa, J. D. McGee, and N. A. Athanasou, "Human osteoclast formation from blood monocytes, peritoneal macrophages, and bone marrow cells," Calcified Tissue International, vol. 62, no. 6, pp. 527-531, 1998.

[27] A. I. Prentice, "Autofluorescence of bone tissues," Journal of Clinical Pathology, vol. 20, no. 5, pp. 717-719, 1967.

[28] P. Karkanas and P. Goldberg, "Phosphatic features," in Interpretation of Micromorphological Features of Soils and Regoliths, G. Stoops, V. Marcelino, and F. Mees, Eds., pp. 521-541, Elsevier, Amsterdam, The Netherlands, 2010.

[29] H. J. Altemüller and B. van Vliet-Lanoë, "Soil thin section fluorescence microscopy," in Soil Micromorphology: A Basic and Applied Science, L. A. Douglas, Ed., pp. 565-579, Elsevier, Amsterdam, The Netherlands, 1990.

[30] K. Nassau, “The causes of color in minerals," Scientific American, vol. 63, pp. 124-154, 1980.

[31] G. McGowan and J. Prangnell, "The significance of vivianite in archaeological settings," Geoarchaeology, vol. 21, no. 1, pp. 93111, 2006.

[32] J. B. Vincent and B. A. Averill, "An enzyme with a double identity: purple acid phosphatase and tartrate-resistant acid phosphatase," The FASEB Journal, vol. 4, no. 12, pp. 3009-3014, 1990.

[33] G. Andersson and B. Ek-Rylander, "The tartrate-resistant purple acid phosphatase of bone osteoclasts-a protein phosphatase with multivalent substrate specificity and regulation," Acta Orthopaedica Scandinavica, vol. 66, supplement 266, pp. 189-194, 1995. 
[34] G. Schenk, L. W. Guddat, Y. Ge et al., "Identification of mammalian-like purple acid phosphatases in a wide range of plants," Gene, vol. 250, no. 1-2, pp. 117-125, 2000.

[35] G. Schenk, N. Mitić, G. R. Hanson, and P. Comba, "Purple acid phosphatase: a journey into the function and mechanism of a colorful enzyme," Coordination Chemistry Reviews, vol. 257, no. 2, pp. 473-482, 2013.

[36] J. M. Halleen, H. Kaija, J. J. Stepan, P. Vihko, and H. K. Väänänen, "Studies on the protein tyrosine phosphatase activity of tartrate-resistant acid phosphatase," Archives of Biochemistry and Biophysics, vol. 352, no. 1, pp. 97-102, 1998.

[37] A. Davies and E. Wilson, "The persistence of seminal constituents in the human vagina," Forensic Science, vol. 3, pp. 45$55,1974$.

[38] F. E. Camps, Medical and Scientific Investigations in the Christie Case, Medical Publications, London, UK, 1953.

[39] H. Borrman, A. Du Chesne, and B. Brinkmann, "Medico-legal aspects of postmortem pink teeth," International Journal of Legal Medicine, vol. 106, no. 5, pp. 225-231, 1994.

[40] P. Sainio, S. Syrjänen, J. P. Keijälä, and A. P. Parviainen, "Postmortem pink teeth phenomenon: an experimental study and a survey of the literature," Proceedings of the Finnish Dental Society, vol. 86, no. 1, pp. 29-35, 1990.

[41] C. Stavrianos, L. Vasiliadis, C. Papadopoulos, O. Pantelidou, K. Tolidis, and P. Dagkalis, "The post-mortem pink teeth phenomenon," Journal of Biological Sciences, vol. 6, pp. 124-127, 2011.

[42] C. W. Van Wyk and B. Werner, "Postmortem pink teeth: histochemical identification of the causative pigment," American Journal of Forensic Medicine and Pathology, vol. 10, no. 2, pp. 134-139, 1989.

[43] C. Ortmann and A. DuChesne, "A partially mummified corpse with pink teeth and pink nails," International Journal of Legal Medicine, vol. 111, no. 1, pp. 35-37, 1997.

[44] C. P. Campobasso, G. Di Vella, A. De Donno, V. Santoro, G. Favia, and F. Introna, "Pink teeth in a series of bodies recovered from a single shipwreck," American Journal of Forensic Medicine and Pathology, vol. 27, no. 4, pp. 313-316, 2006.

[45] M. M. Mitchell, J. G. Barabe, and A. B. Quandt, "Chicago's 'archaic Mark' (ms 2427) II microscopic, chemical and codicological analyses confirm modern production," Novum Testamentum, vol. 52, no. 2, pp. 101-133, 2010.

[46] A. Quandt, "Preserving the archimedes palimpsest," in The Archimedes Palimpsest. I Catalogue and Commentary, R. Netz, W. Noel, N. Tchernetska, and N. Wilson, Eds., pp. 129-171, Cambridge University Press, Cambridge, UK, 2011. 


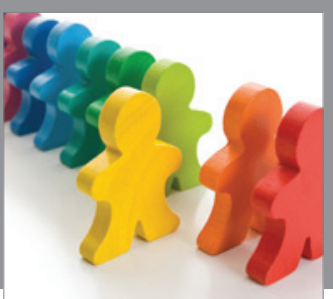

Autism

Research and Treatment
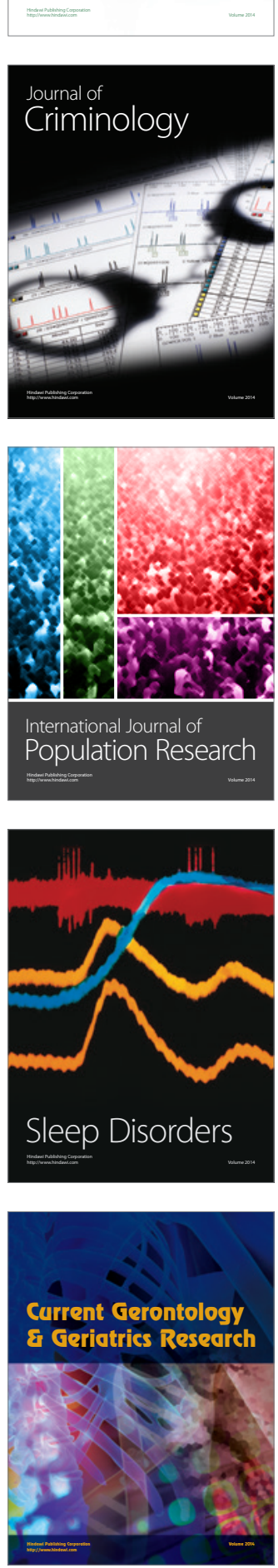

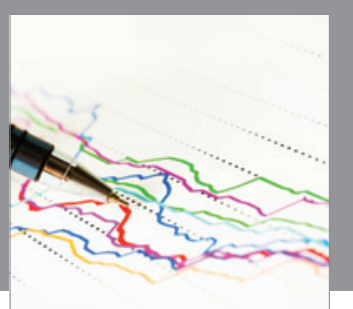

Economics

Research International
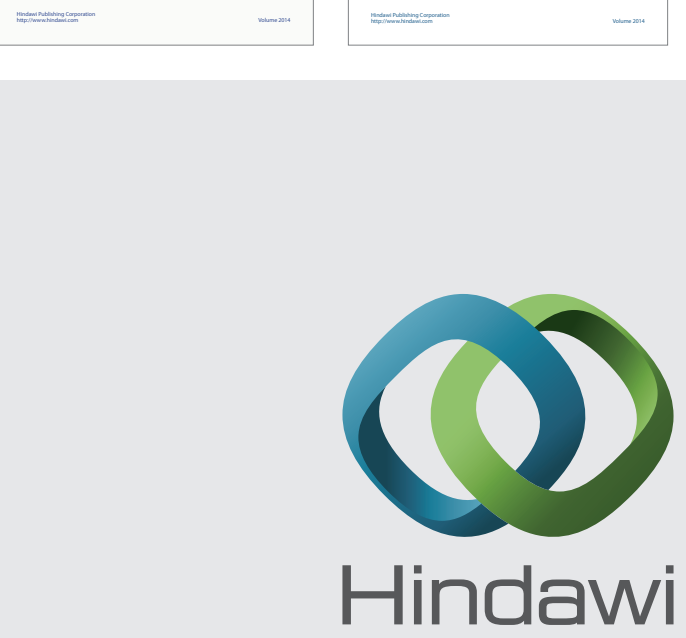

Submit your manuscripts at

http://www.hindawi.com
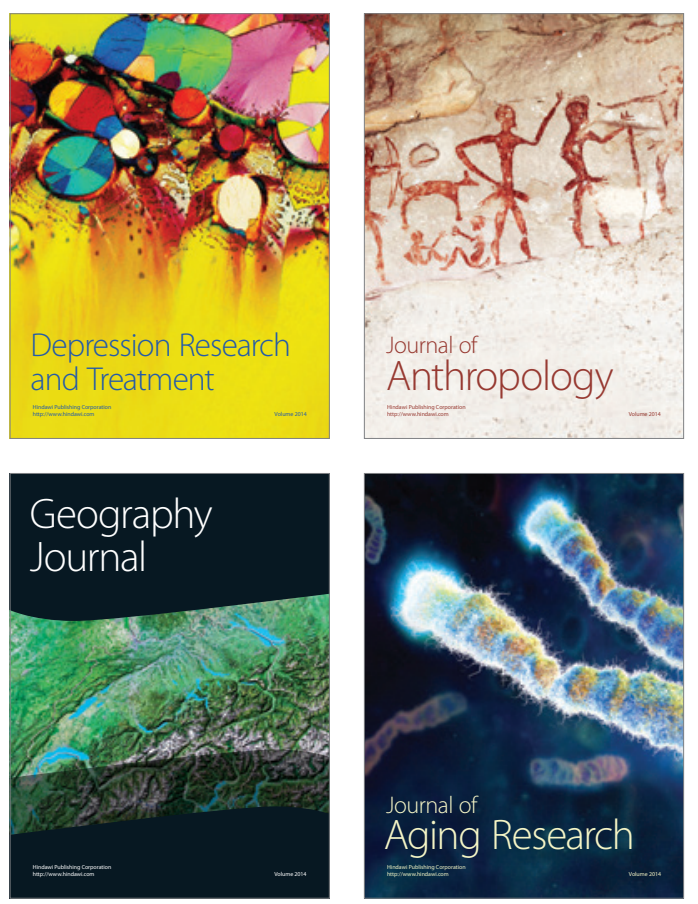
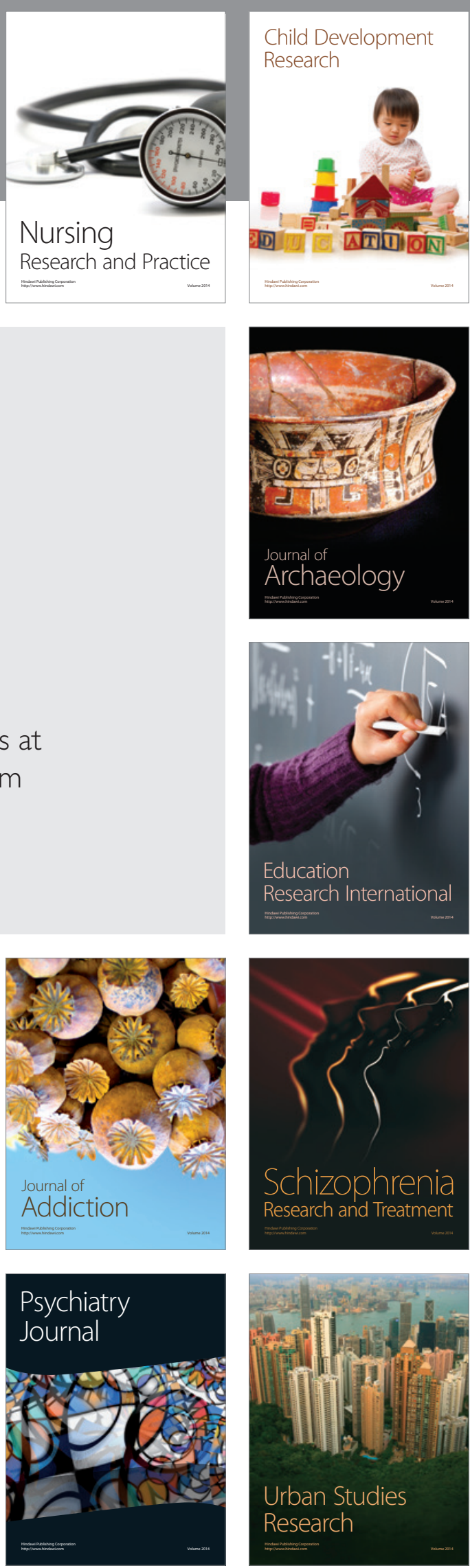